\title{
SeaTouch: \\ A haptic and auditory maritime environment for non visual cognitive mapping of blind sailors
}

\author{
Mathieu Simonnet ${ }^{1 \star}$, Dan Jacobson ${ }^{2}$, Stephane Vieilledent ${ }^{1}$, and Jacques \\ Tisseau $^{1}$ \\ ${ }^{1}$ European Center for Virtual Reality (CERV), Britain European University (UEB) \\ 25 rue Claude Chappe, 29280 Plouzane, France. \\ \{mathieu.simonnet, stephane.vieilledent\}@univ-brest.fr, \\ jacques.tisseau@enib.fr \\ http://www.cerv.fr \\ ${ }^{2}$ Investigating Multi Modal Representations of Spatial Environments (IMMERSE), \\ Department of Geography, University of Calgary \\ 2500 University Drive NW, Calgary, AB, T2N 1N4, Canada. \\ dan.jacobson@ucalgary.ca, \\ http://www.immerse.ucalgary.ca
}

\begin{abstract}
Navigating consists of coordinating egocentric and allocentric spatial frames of reference. Virtual environments have afforded researchers in the spatial community with tools to investigate the learning of space. The issue of the transfer between virtual and real situations is not trivial. A central question is the role of frames of reference in mediating spatial knowledge transfer to external surroundings, as is the effect of different sensory modalities accessed in simulated and real worlds. This challenges the capacity of blind people to use virtual reality to explore a scene without graphics. The present experiment involves a haptic and auditory maritime virtual environment. In triangulation tasks, we measure systematic errors and preliminary results show an ability to learn configurational knowledge and to navigate through it without vision. Subjects appeared to take advantage of getting lost in an egocentric "haptic" view in the virtual environment to improve performances in the real environment.
\end{abstract}

Key words: Navigation, spatial frames of reference, virtual reality, haptic, blind, sailing.

\section{Introduction}

Since Vygotsky's "theory of mediated activity" [1], the psychological community has paid particular attention to psychological tools. For example, in the spatial domain maps and compasses provide support for humans to think about

\footnotetext{
* Currently a postdoctoral fellow at the Naval Academy Research Institute in LanvocPoulmic, France.
} 
space. Thorndyke and Hayes-Roth [2] revealed that map learning is superior to environmental navigaton for judgements of relative locations and straight line distances among objects. Thus, learning a configurational layout does not necessarily require entire body displacement. Clearly, there are many differences between consulting a map and walking in an environment. While the map consultation unfolds in an allocentric spatial reference frame, independent of the perceiver position and orientation, the walking sequence takes place in an egocentric frame of reference which is directly relative to the current state of the body [3]. Thinus-Blanc and Gaunet [4] showed the necessity to coordinate these two views using invariants as a form of mediated common frame of reference.

The development of virtual reality techniques have provided researchers with promising tools to investigate how individuals learn space in controlled environments. Tlauka and Wilson [5] compared the spatial knowledge of subjects after a computer simulated navigation in an egocentric reference and the consultation of a map in an allocentric reference. Referring to the results found by Thorndyke and Hayes-Roth [2], the authors concluded that virtual and real navigation lead to the potential for building equivalent spatial knowledge. Similar outcomes were found by Richardson, Montello and Hegarty [6]. However, most of the time, during navigation in virtual environments, subjects predominantly accessed only visual information. This is in contrast to environmental navigation where increased acces to visual cues and displacements of the entire body provide people with inertial, kinesthetic and proprioceptive information. However, numerous studies from spatial virtual environments found that learning large-scale environments could be done effectively from purely visual sources and did not require body based information at all $[7,8]$.

As vision is the spatial sense par excellence and virtual environments are both predominantly visual and predicated by the use of vision, the utility and the ability of blind people to generate meaningful spatial information via exposure to virtual environments has not been widely explored (See Lahav and Mioduser [9] for an exception). Consequently the question of what the role of virtual environments are able to play as a tool to aid the spatial knowledge learning of blind people remains largely unknown. For centuries there have been philosophical and experimental debates as to the capacity of people without sight to acquire a functional and holistic view of geographic space (See Ungar [10] for a review). The "difference theory" contends that blind people have no deficit in spatial processing but need more time to develop it [11]. The difficulties experienced by blind individuals in traversing an environment derive in part from their perceptual inability to gather distal (out of touch) spatial information [12]. While micro navigation, that which is proximal to the body, is taught as obstacle avoidance in traditional orientation and mobility training, and the environmental learning of an area is most commonly acquired through sequential and procedural exploration. The generation of configurational (macro level) knowledge of an environment remains problematic and is only achieved through intensive exploration of an environment $[13,14]$. However, spatial learning can be facilitated by the use of other spatial representations as tactile maps and 
models [15], digital touch and auditory interfaces to information [13,16], haptic and auditory displays [17] or personal guidance systems $[18,19]$. In any case, the ability to integrate, translate and move between experiences and representations relative to the egocentric and allocentric spatial frames of reference critical.

Blind people are able to successfully and independently navigate urban environments, where necessary using long canes and guide dogs as obstacle avoidance tools at the micro level. Access to the tools listed above is rare at best [20,21], however the acquistion of route, procedural and landmark knowledge, remains possible, providing enough information for blind people to locate and orientate themselves relative to the surroundings. In a built environment, there is a wide array of environmental cues, for example, sidewalks, road edges and junctions, auditory and olfactory landmarks, that facilitate the generation of spatial knowledge of an area and the ability to recreate routes. The guideing question behind the research is the complex issue of how do blind individuals locate themselves in natural environment that does not provide conventional urban cues for navigation, or the ability to spatially update through vestibular, kinaesthic, proprioceptive or inertial processes [22]. With an environmental tabula rasa, unable to access the cues above how would blind individuals be able to locate, orient and comprehend their spatial environmnet. Such an environment is provide by the ocean. Here, are the use of tactile maps and vocal compasses sufficient to connect egocentric and allocentric frames of reference?

In Brest (France), blindsailors were able to helm the sailboat in a straight line due to wind sensations [23]. In this case, the wind direction appeared to be a key feature to hold one's course and became the main directional reference. However, avoiding distant obstacles like rocks and locating themselves on the map remains a complex spatial task for blind sailors. In a maritime environment the type and variability of potential environmental information available is very different from a land based situation. These include, wind (speed and direction), boat displacement (speed, heading, pitch, roll, yaw), and potentially spatial updating via path integration based upon monitoring tacking, from a combination of the above information. All of this information is spatially and temporally highly variable. In order to assess these questions and in an applied manner to provide a navigational solution, we developed "SeaTouch". This application allows blind people to explore a representation of a maritime environment by means of a haptic and auditory interface. This enables virtual navigation with egocentric and allocentric "haptic views". Practically, this system aims to help blind people to master sailing navigation during real voyages, and in this manner is analogous to a land based personal guidance system [19].

After the description of the SeaTouch functionalities, we present an experiment to compare the performances obtained by blind sailors after virtual training sessions perfomed in egocentric or allocentric conditions. 


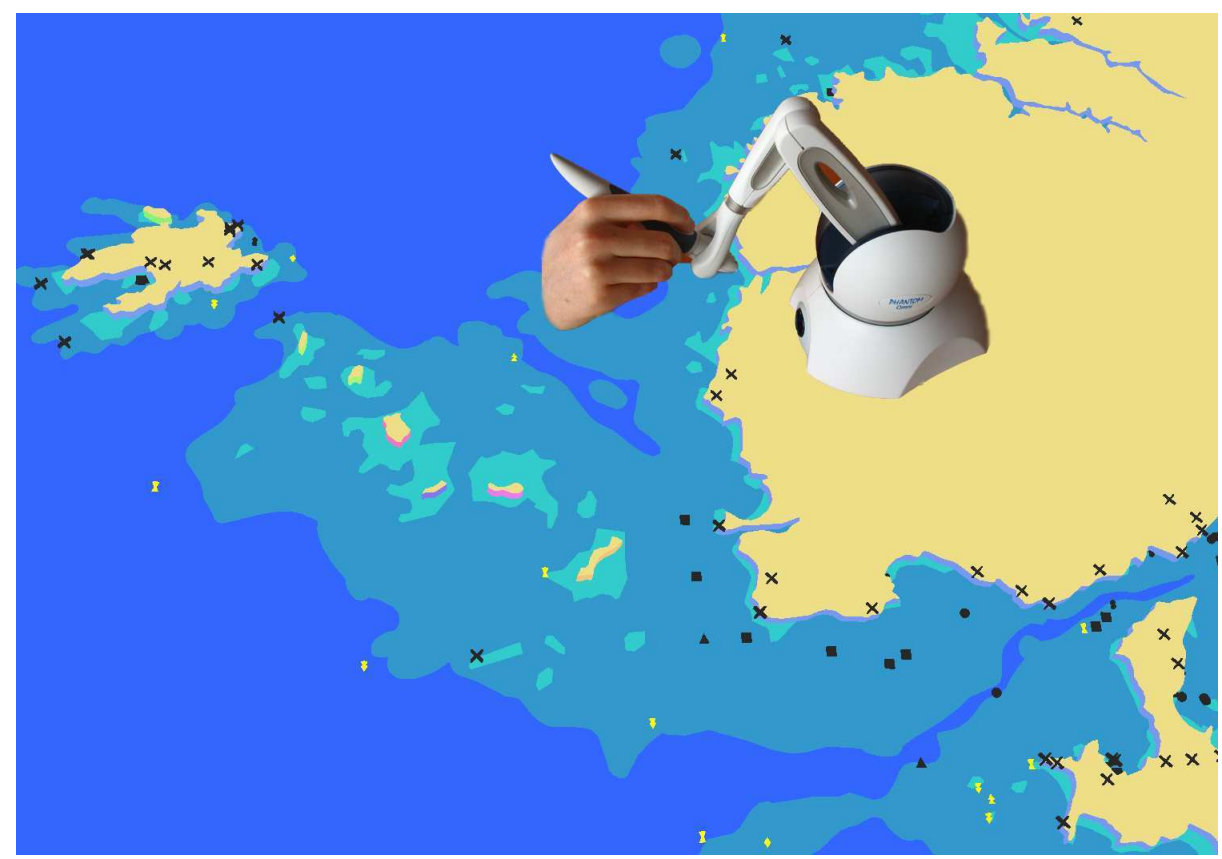

Fig. 1. A visualization of the SeaTouch virtual maritime environment. A participant's hand is interacting with the stylus of the Phantom haptic mouse. The land area, coastline, and maritime features are displayed. 


\section{SeaTouch}

\subsection{General Description}

SeaTouch software and hardware aim to provide for blind people's cartographic needs, in a maritime environment using haptic sensations, vocal announcements and realistic sounds (Fig. 1). SeaTouch allows blind sailors to prepare their maritime itineraries. The digital maritime charts used in the development of SeaTouch conform to the S-57 International Hydrographic Office (IHO) exchange format, ensuring opportunities for interoperability. The digital charts contain many relevant geographic objects; "Handinav" software was developed to transform the S-57 data into XML structured files. Thus, objects of particular salience can be chosen to be displayed or not: sea areas, coastlines, land areas, beacons, buoys, landmarks were used in our research. Additional data contained in this maritime XML format is retained for potential future use. The position of the boat can be selected by entering coordinates in the simulator when it is started. The simulated weather conditions, such as the direction and the speed of the wind are modifiable. When simulation is on, the speed of the boat results from the interaction of the direction and speed of the wind with the orientation of the boat, generating a new heading speed. These calculations are based upon ocean based data collection, from an 8 meter sailboat "Sirius". Blind sailors choose the boat's heading during the entire simulation by using the right and left arrows of the computer keyboard. When the boat hits the coast, the simulation stops, this is indicated to the users via an auditory "crash".

\subsection{Haptic Contacts and Constraints}

The representational workspace is in the vertical plane, 40 centimetres wide, 30 centimetres high and 12 centimetres deep. Using a Phantom Omni force-feedback device, via a haptic cursor, calibrated to the representational workspace, blind participants explore the scene (Fig. 1). They touch different objects on the maritime maps as 2D-extruded haptic features. The salient features are sea surface, coastline, land area, navigational beacons, buoys and landmarks. The sea surface and land area are formed by two flat surfaces separated by two centimetres. Between the land and sea, the coastlines form a perpendicular wall, analogous to a cliff face, that allows users to follow it with the Phantom. The display of coastlines uses the "contact haptic force feedback", a virtual wall. By contrast, for beacons, buoys and landmarks, we apply a "constraint haptic force feedback" to a spring of one centimeter diameter. This spring is an active force feedback field that maintains the cursor inside of the object with a 0.88 Newton force, analogous to a "gravity well". In order to move outside of the spring, participants have to apply a stronger force. The position of the boat is displayed by the same haptic spring effect. It can be located from anywhere in the workspace by pressing the first button of the Phantom stylus, then the haptic cursor is relocated to the current position of the boat. 


\subsection{Sonification}

In the sonification module, as soon as the stylus is in contact with virtual geographic objects audible naturalistic sounds are played. When in contact with the sea, a looping water sound is played. When the haptic stylus is touching the coastline, a virtual cliff face, the sounds of seabirds are played, and when land areas are in contact with the stylus the sounds of land birds are played. Prior testing confirmed clear discriminability of the sea and land birds sonification signals. If participants push through the sea surface, they hear the sound that a diver would make. If the cursor is wandering in the air, a wind sound is played. It is possible to touch the wake of the boat by hearing wash sounds. Our intention is that the redundancy and overlap between haptic and auditory information make this virtual environment as intuitive as possible.

\subsection{Vocalization}

Using "Acapela" vocal synthesis, a text to speech software, auditory information can be automatically spoken by SeaTouch. When the Phantom cursor enters in a beacon, buoy or landmark field, the nature and the name of these are announced. Alternatively, blind participants can ask for information about distances and directions between the boat and the beacons or between two beacons. The format of this information can be preselected by the user, distance in nautical miles, or in kilometers, or in time relative to the current speed of the boat. In the allocentric representation mode, directions can be vocalized in the cardinal system (North, South, etc.) or in numeric cardinal degrees $(0-360)$. In the egocentric representation mode directions can be obtained in hours relative to the boat orientation (noon is in front of the boat and 6 o'clock is behind), or in port and starboard numeric degrees relative to the boat orientation. For instance 90 degrees to starboard would be equivalent to 3 o'clock.

\subsection{Virtual Navigation and Spatial Frames of Reference}

SeaTouch software allows virtual interaction with two modes of haptic perspective : the allocentric ("bird's eye view") or the egocentric ("on board view") perspectives.

Northing / allocentric mode. The northing view provides a conventional presentation format of the scene, where the map remains invariant in a fixed frame of reference, aligned north up. The subject faces the north and the boat moves over the map. Thus exploration unfolds in an allocentric frame of reference (Fig. 2).

Heading / egocentric mode. By contrast, the heading view takes place in an egocentric frame of reference. Although the participant remains in an orthographic map view the participants' view of the map is continually re-orientated to always face the heading of the ship aligned to the top of the scene. This means that the ship does not rotate in the workspace, but the map rotates to maintain the 
heading of the ship to the top of the scene. Thus, the scene is dynamic and shifts as the blind sailor explores the auditory haptic representation (Fig. 3). In this condition the Phantom is comparable to a sort of "long maritime white cane" that the subject can use either to touch the boat itself or to localize the obstacles in the scene. The scale of the scene clearly facilitates exploration beyond the proximal in the micro environment, affording a "map-like" overview of the area.
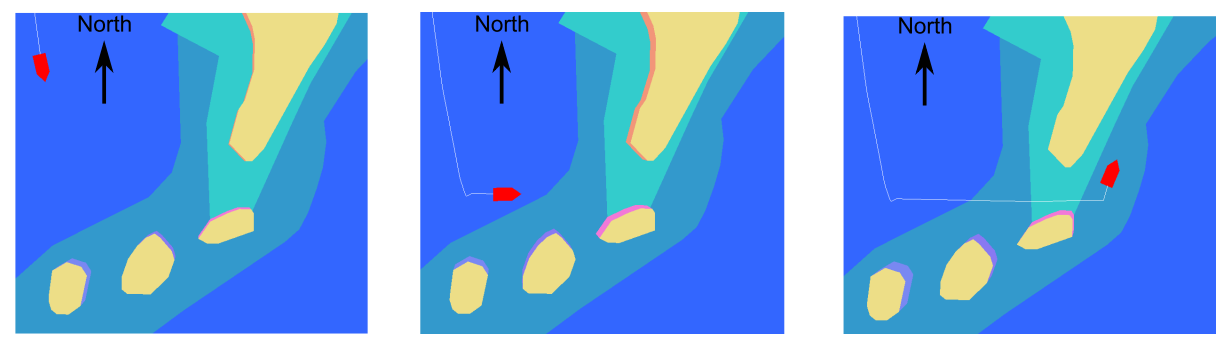

Fig. 2. The northing mode (allocentric) of SeaTouch: while changing boat directions, the boat moves on the map but the orientation of the map stays stable.
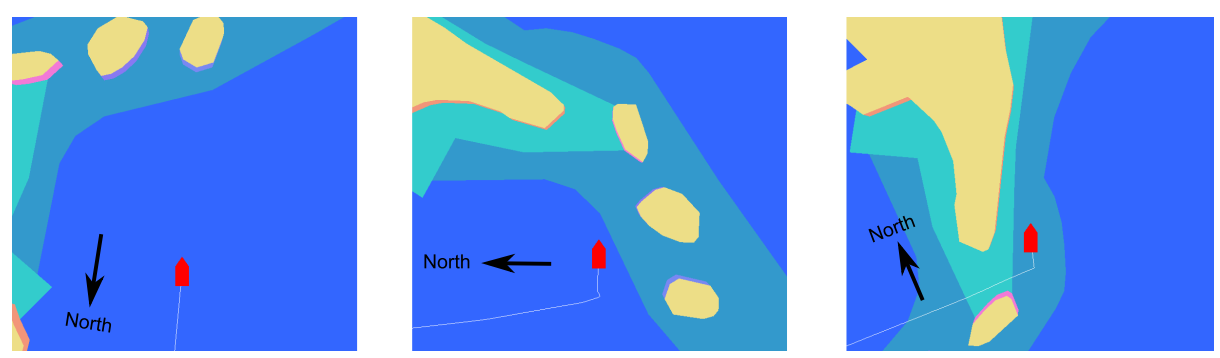

Fig. 3. The heading mode (egocentric) of SeaTouch: while changing boat directions, its position and orientation in the workspace stay stable but the map orientation moves to ensure a "boat up" view.

Aim of the study. The heading and northing possibilities offered by SeaTouch to navigate in a virtual environment raise the question of their respective impact to the construction of an efficient non visual spatial representation at sea. The research focuses on investigating the difference between information gained in either of these frames of reference and the participants ability to transfer this information to a sailing activity in the ocean environment. Ultimately assessing whether virtual navigation in a heading condition (egocentric) is more efficient 
that in a northing condition (allocentric) to help facilitate blind sailors to train, locating the landmarks, or beacons, in the environment during a real voyage.

\section{Method}

\subsection{Subjects, Cartographic Material and Experimental Organization}

Subjects. As a preliminary study two blind sailors performed our experiment. They both navigate at sea regularly, are familiar with maps and computers.

Cartographic Material. During the learning tasks of each condition, the subjects were asked to explore SeaTouch maps including a configuration of six named beacons to set up their itinerary. This was composed of five ordered directions between these named anchor points (e.g. "rock" "raft", "spot", "pole", "net", "buoy"). The configuration of the points are the same in both conditions (egocentric and allocentric) except that we applied a mirroring symmetry translation along the north-south axis and changed the names of the beacons to avoid any learning effects (Fig. 4). In addition subjects performed the tasks of the two conditions in opposite orders, in a cross over design.

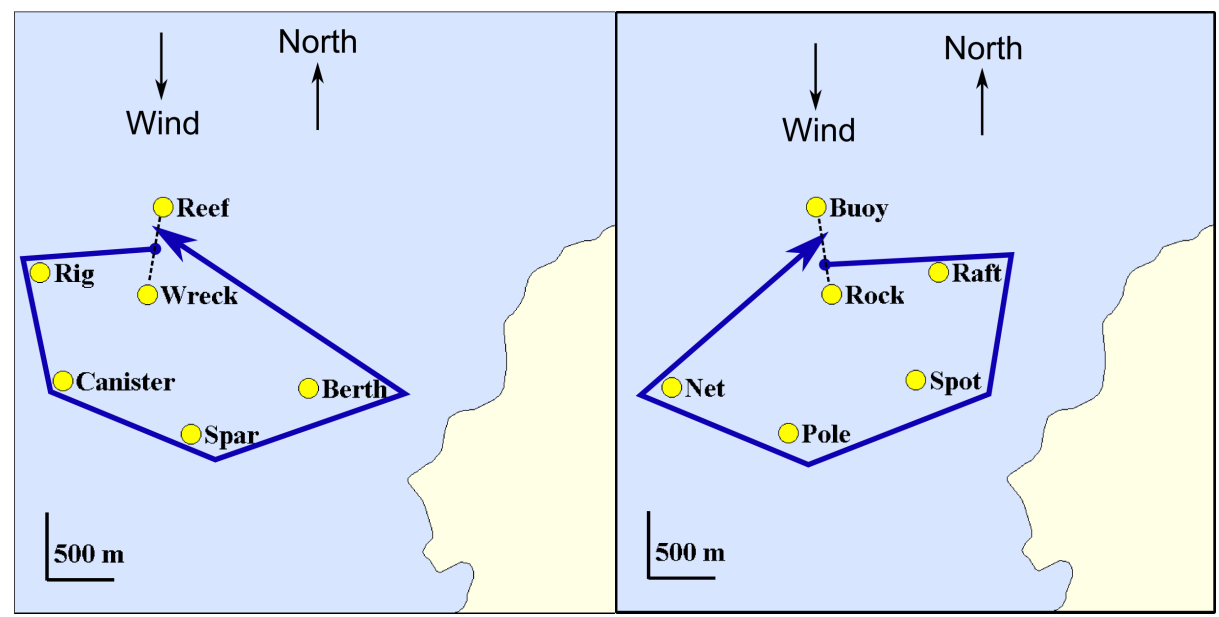

Fig. 4. Northing and heading maps. On the left the map we used in the northing condition, on the right the one we used in the heading condition. These two maps are similar except that, for illustrative purposes a symmetric transformation was applied to the right map relative to the vertical axis crossing the central point. The dark blue lines represent the trajectories of each course. 
Experimental organization (Fig. 5). In order clarify each condition (heading versus northing), the virtual learning task can be divided in two sub-learning phases. The first phase consisted in exploring the map in a static setting; the participants familiarized themselves with the equipment, the interface and its operation until they were satisfied, and then they acquired their itinerary. The second learning phase was made up of navigating through the virtual environment attempting to follow their itinerary between the named anchor points. Then, after completing these two sub-learning tasks that we describe more precisely below, subjects had to actualize their learning by navigating in the open sea evaluation task depicted in further below (See subsection 3.3).

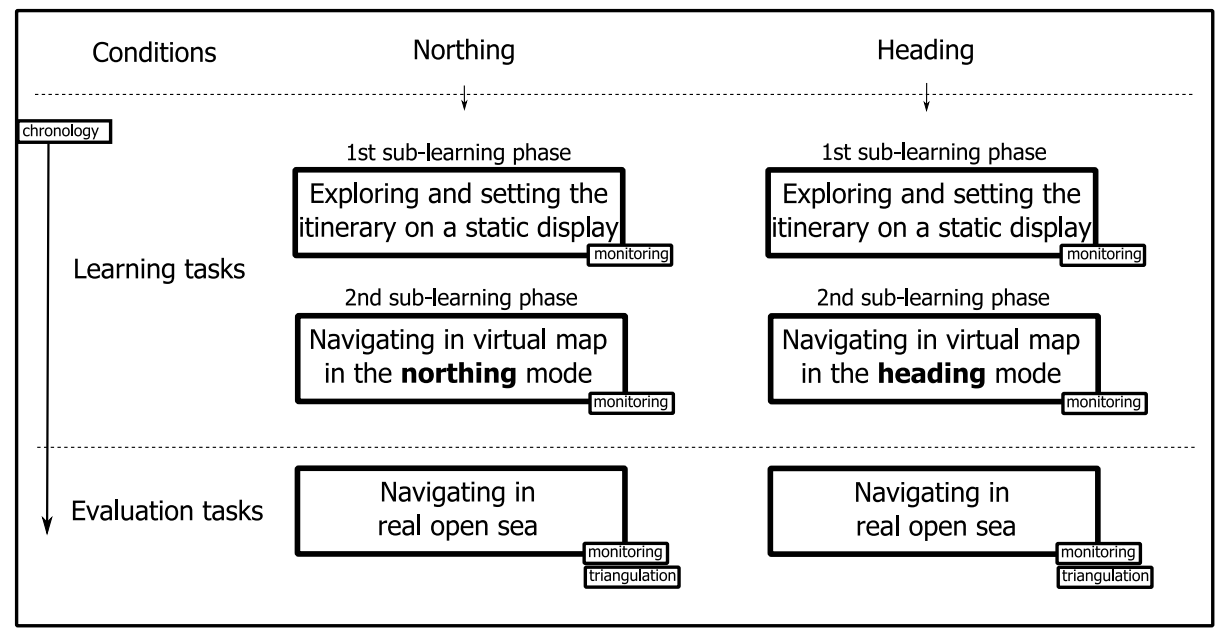

Fig. 5. The experimental organization.

\subsection{Learning Task}

In each condition, during the first sub-learning phase, the subjects explored the virtual map without navigating. They had to determine the five bearings and distances necessary to cross the departure line, turn around the beacons and return to cross the arrival line which is actually the same as the departure line (Fig. 4). In northing (allocentric) condition, the departure/arrival line is located between the "buoy" and the "raft" and the subject had to traverse the beacons on the starboard, the right, in a clockwise direction. In the heading (egocentric) condition, the departure/arrival line is located between the "reef" and the "wreck" and the subjects had to traverse the beacons to port, the left, in a counter-clockwise direction (Fig. 4).

Itinerary Setting. In a first sub-learning phase (Fig. 5), the establishment of the bearings and the distances of the itinerary of the course were done by using the 
"measure command" of SeaTouch. Here, subjects placed the haptic cursor to an initial point and said "origin" in the microphone, a voice answered "origin" Then subjects moved the cursor to a destination point and said "measure". The voice answered them the bearing (in cardinal numeric) and distance (in kilometers) between these two points. They set the all itineraries in this manner. This learning sub-task is exactly the same in the both conditions except for the positions of the landmarks.

Virtual Navigation. By contrast, in a second sub-learning phase (Fig. 5), subjects performed a virtual navigation in a heading or northing mode respectively corresponding to the egocentric and allocentric conditions. Here they could actively direct the orientation of the ship by speaking in increments of 1 or 10 up to 90 degrees to starboard or to port in the microphone. The speed of the boat only depended of its interaction with the wind. This blew from the north. If the angle between the ship direction and the wind orientation was less than 45 degrees, it could not proceed, replicating conditions in the open water. During this virtual navigation, blind sailors could ask for the bearings (in cardinal numeric degrees) and the distance (in kilometers) of the nearest beacon by saying "beacon". They also could ask for the current speed and heading. During the virtual navigation, we recorded the exploration displacements of the haptic cursor and of the trajectory of the boat. We called this record the monitoring (Fig. 5).

This procedure was replicated with the participants repeating the experiment in the alternate condition, that is participant who explored in the heading (egocentric) mode now explored the virtual environment in the northing (allocentric) mode and vice versa.

\subsection{Evaluation Task}

Here, we assessed how precisely subjects were able to locate themselves on the sea when navigating aboard a real sailboat encountering all of the usual constraints of sailing, such as tacking. After the virtual navigation, the blind sailors navigated in an open ocean environment aboard Sirius, an 8 meters long sailboat of the Orion association. The wind also blew from the north ( \pm 15 degrees). Subjects were asked to follow their set of named beacons, gathered from the virtual explorations in SeaTouch. In the real navigation, they managed Sirius by the same commands (1, 10 or 90 degrees) which were applied to an "automatic pilot". The automatic pilot is an electrical system, including an electronic compass, that movers the tiller to maintain a magnetic heading of the boat without assistance. In essence the participants were sailing "hands free", but encountering the complete array of other ocean based cues, such as, wind, swell and the roll, pitch and yaw of the boat. As in virtual navigation, subjects could ask information about the nearest beacon, the speed and heading of the sail boat. However, they did not have the haptic interface at their disposal so had no access to other distal cues.

To assess how precisely blind sailors could locate themselves, we asked them to point out the directions of three beacons in the middle of each of their five 
segments of the itinerary and we picked up these directions with a bearing's compass. So, we obtained fifteen angular errors about the directions of the landmarks. Directional pointing data was used to apply the projective convergence technique [24]. This triangulation provided us with five error triangles. Their areas informed us about the consistency of the responses (Fig. 6).

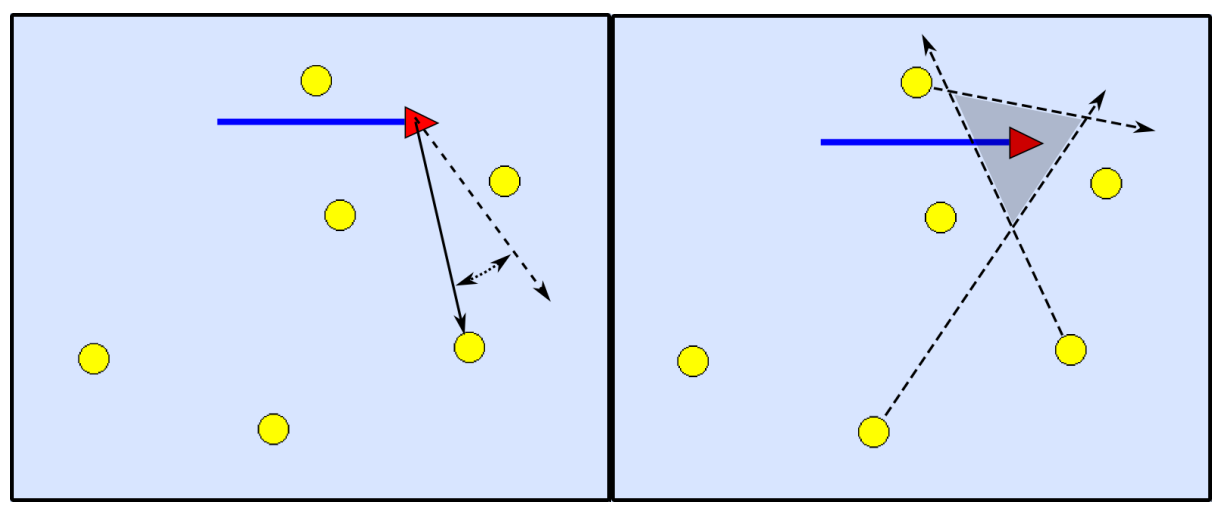

Fig. 6. Examples of angular error (on the left) and error triangle (on the right). The yellow circles are the beacons. The small red triangle is the sailboat. The large blue line is the track of the ship. The dotted lines are the directions estimated. On the left figure, the angle between the plain and dotted lines constitutes the angular error. On the right figure, we report estimations from the beacons to the ship. Where the dotted lines cross, the large grey triangle drawn is the error triangle.

\section{Results}

Due to the non normal distribution and measurement level of the data we used the non parametric Wilcoxon paired test to evaluate differences in the egocentric and allocentric frames of reference.

The comparison of the angular errors of subject 1 after performing virtual training in heading (egocentric) and northing (allocentric) conditions revealed a significant difference $(\mathrm{p}=0.02)$. After heading virtual training, 14 responses among 15 were under 30 degrees of error (Fig. 7). After northing virtual training, only 8 estimations were under 30 degrees. So, the angular errors of subject 1 were significantly less important in the heading condition. By contrast the comparison of the angular errors of the subject 2 in heading and northing conditions did not reveal any significant difference $(\mathrm{p}=0.82)$ (Fig. 7$)$.

These results were confirmed when we compared the areas of error triangles. For subject 1, all areas of error triangles were under $0.4 \mathrm{~km}^{2}$ after virtual training in heading condition. By contrast, after virtual training in northing condition, 3 responses among 5 were over $0.5 \mathrm{~km}^{2}$. So, relative to the areas of the error 

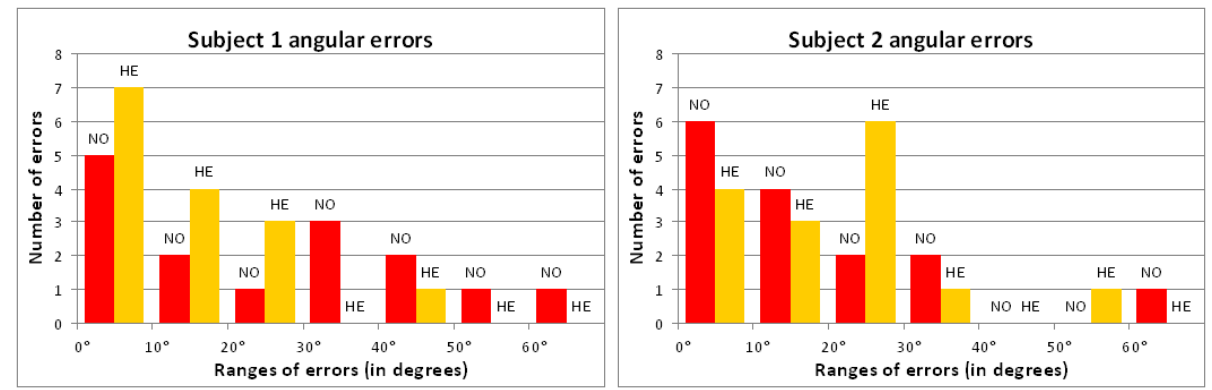

Fig. 7. Range of angular errors of the subjects 1 and 2 at sea after training in northing (NO) and heading (HE) conditions.

triangles, subject 1 performed significantly $(\mathrm{p}=0.04)$ better at sea after training in heading (egocentric) condition (Fig. 8). Subject 2, replicating the situation for angular errors, no significant difference was found between the error triangles in northing and heading conditions.

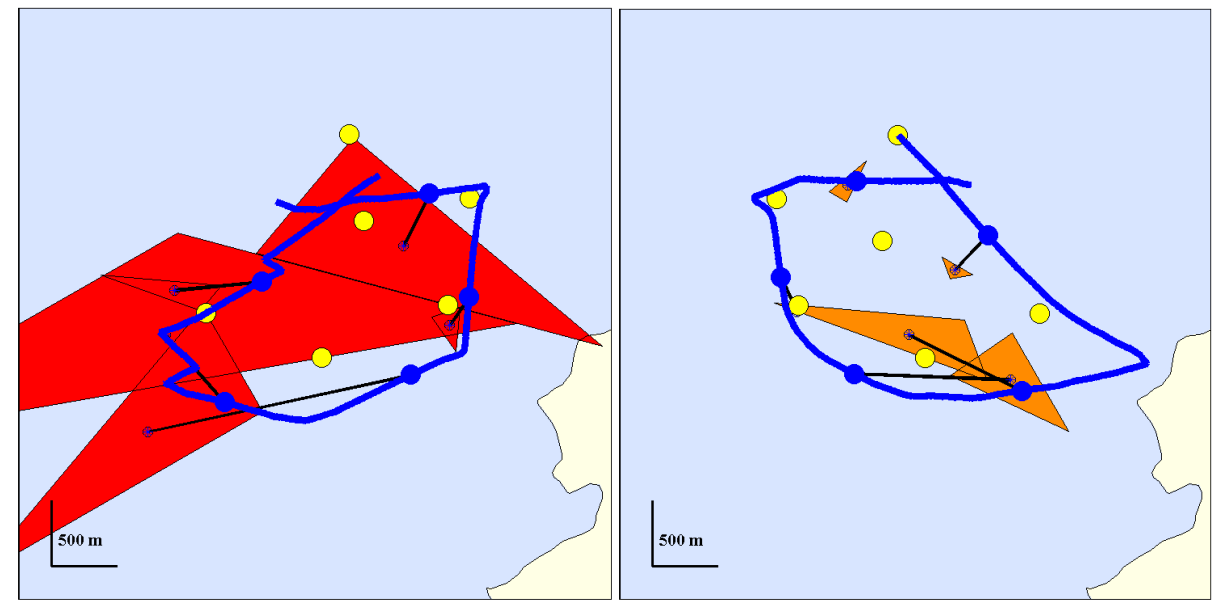

Fig. 8. Graphic representation of the trajectories and the errors triangles of subject 1 at sea after a virtual training in a northing mode (on the left) and in a heading mode (on the right).

The better performances of subject 1 could not come from the learning effect because he firstly performed the heading condition. So these results are reinforced by the order of the experiment. The qualitative results provided with the monitoring allow us to see that the exploration movements of subjects 1 and 2 were different, especially in heading condition (Fig. 9). This report suggests an interesting discussion point, as to what is the role of "getting lost"? In other 
words what are the effects and differences in acquiring levels of spatial knowledge, does exploring an environment searching for information necessarily lead to higher more configural spatial knowledge rather than following a pre-learned prescribed route.
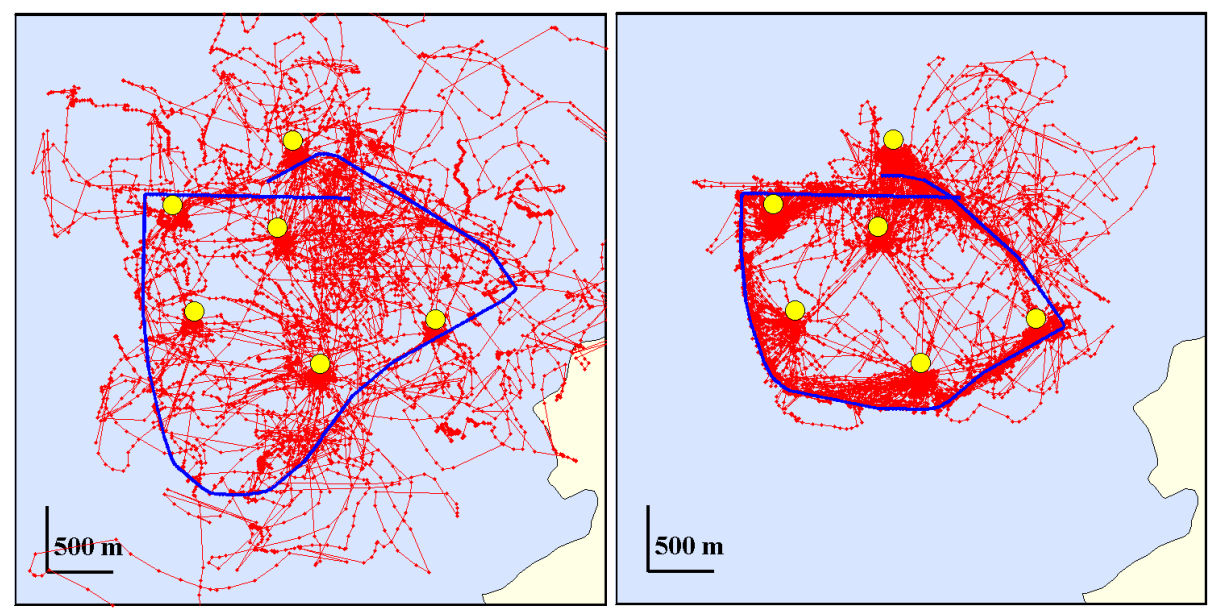

Fig. 9. The heading haptic patterns of exploration. On the left is the recorded pattern of exploration of the subject 1 . On the right is the recorded pattern of exploration of the subject 2 .

\section{Discussion}

The present study aimed at assessing the influence of egocentric and allocentric representations in virtual training, and their effect on participants abilities to locate and navigate in a true maritime environment without vision. The evaluation task was performed during true navigation that obviously took place in an egocentric spatial frame of reference. An explanation of the better results of subject 1 in the heading (egocentric) condition could be that the transfer of spatial knowledge is favored by the similarity of the spatial frames of reference involved in the learning and evaluation tasks. In this case, it would be reasonable to suppose that the lack of significant difference between the results obtained by the subject 2 after heading and northing virtual training could come from the learning effect. As we mentioned before, the second subject first trained in heading condition. So, the potential benefits of this condition could be neutralized by the learning undertaken by the repetition of the experiment.

However, the qualitative analysis of the exploratory strategies in virtual environment suggested another explanation. Actually, it seems that subject 1 often explored in directions which were out of the set up itinerary (Fig. 9). One could 
reasonably propose that this subject got often lost, or traveled significantly "off route". These kinds of movements did not appear on the subject 2 exploration pattern. This subject seems to follow regularly the sequence of named beacons. This difference could be the key to our results. Getting lost corresponds to a rupture between the actual egocentric spatial perception and the previously more global spatial representation which can be considered as allocentric [25]. So, when subject 1 got lost, he had to mentally try and connect the actual nearest beacon direction with the configuration of the anterior memorized map. This cognitive process could be an efficient training mechanism if we refer to Thinus-Blanc [4] who showed that mastering space consists in coordinate egocentric and allocentric spatial frames of reference using the concept of invariant. For these authors, an invariant would be a common entity of the egocentric field of perceptions and the allocentric spatial representation. In this case, subject 1 had to mentally identify an invariant when getting lost in the virtual world whereas subject 2 did not need to process this spatial reasoning because he did not encounter any difficulties in following the itinerary previously learned. This could explain why subject 2 did not benefit from the heading navigation.

This small prelimary case study shows that it could be beneficial to get lost in a virtual environment in an egocentric view in order to practice connecting egocentric and allocentric frames of reference. Our results should be interpreted with caution due to the small sample size $(n=2)$, and the inherent possibility for a large role to be played by individual differences. Inspite of this small sample size the data collected are able to demonstrate that clearly different strategies may be utilised by people using the SeaTouch. However the experimental structure and methodology used is able to provide useful, insightful information. Our results highlight the need for further investigations with more subjects. In more general terms we have an environment and methodolody for exploring the role of egocentric and allocentric, haptic and auditory learning in virtual environments for people without vision and the transfer of this knowlegde to maritime surroundings. This affords us the opportunity to investigate further the role of frames of reference, map alignment, and a user's perspective on spatial information, in combination with issues of multimodal spatial data representation. The role of each of these factors transcends beyond the maritime or vision impaired community and are central to understanding how we learn from virtual environments and then utilise this information in the real world.

\section{References}

1. Vygotsky, L.S.: Mind of society. Harvard University Press, Cambridge, MA (1930)

2. Thorndyke, P., Hayes-Roth, B.: Differences in spatial knowledge acquired from maps and navigation. Cognitive Psychology. 14, 560-589 (1982)

3. Klatzky, R.: Allocentric and egocentric spatial representations: Definitions, distinctions, and interconnections. In: Freksa, C., Habel C., Wender, K.F. (eds.), Spatial cognition - An interdisciplinary approach to representation and processing of spatial knowledge. Lecture Notes in Artificial Intelligence, vol. 1404, pp. 1-17, SpringerVerlag, Berlin (1998) 
4. Thinus-Blanc, C., Gaunet, F.: Representation of space in blind persons: vision as a spatial sense?. Psychological Bulletin. 121(1), 20-42 (1997)

5. Tlauka, M., Wilson, P.: Orientation-Free Representations from Navigation through a Computer-Simulated Environment. Environment and Behavior. 28(5), 647-664 (1996)

6. Richardson, A., Montello, D., Hegarty, M.: Spatial knowledge acquisition from maps and from navigation in real and virtual environments. Memory and Cognition. 27(4), 741-50 (1999)

7. Rossano, M., West, S., Robertson, T., Wayne, M., Chase, R.: The acquisition of route and survey knowledge from computer models. Journal of Environmental Psychology. 19(2), 101-115 (1999)

8. Waller, D., Greenauer, N.: The role of body-based sensory information in the acquisition of enduring spatial representations. Psychological Research. 71(3), 322-332 (2007)

9. Lahav, O., Mioduser, D.: Haptic-feedback support for cognitive mapping of unknow spaces by people who are blind. International Journal of Human-Computer Studies. 66, 23-35 (2008)

10. Ungar, S.: Cognitive mapping without visual experience. In: Kitchin, R. and Freundschuh, S. (eds), Cognitive Mapping: Past, Present and Future, pp 221-248, Routledge, London (2000)

11. Kitchin, R., Blades, M., Golledge, R.: Understanding spatial concepts at the geographic scale without the use of vision. Progress in Human Geography. 21(2), 225-242 (1997)

12. Millar, S.: Models of Sensory Deprivation: The Nature/Nurture Dichotomy and Spatial Representation in the Blind. International Journal of Behavioral Development. 11(1), 69-87 (1988)

13. Jacobson, D.: Cognitive mapping without sight: Four preliminary studies of spatial learning. Journal of Environmental Psychology. 18, 189-305 (1998)

14. Jacobson, R., Lippa, Y., Golledge, R., Kitchin, R., Blades, M.: Rapid development of cognitive maps in people with visual impairments when exploring novel geographic spaces. Bulletin of People-Environment Studies. 18, 3-6 (2001)

15. Casey, S.: Cognitive mapping by the blind. Journal of Visual Impairment and Blindness. 72, 297-301 (1978)

16. Jacobson, R.D.: Representing Spatial Information Through Multimodal Interfaces: Overview and preliminary results in non-visual interfaces. In: 6th International Conference on Information Visualization: Symposium on Spatial/Geographic Data Visualization, pp. 730-734. IEEE Proceedings, London (2002)

17. . Rice, M., Jacobson, R.D., Golledge, R.G., Jones, D.: Design Considerations for Haptic and Auditory Map Interfaces. Cartography and Geographic Information Science. 32(4), 381-391 (2005)

18. Golledge, R.G., Loomis, J.M., Klatzky, R.L., Flury, A., Yang, X.L.: Designing a personal guidance system to aid navigation without sight: Progress on the GIS component. International Journal of Geographical Information Systems. 5, 373-396 (1991)

19. Golledge, R.G., Klatzky, R.L., Loomis, J.M., Speigle, J., Tietz, J.: A geographical information system for a GPS based personal guidance system. International Journal of Geographical Information Systems. 12, 727-749 (1998)

20. Rowell, J., Ungar, S: The world of touch: an international survey of tactile maps. Part 1: production. British Journal of Visual Impairment. 21(3), 98-104 (2003)

21. Rowell, J., Ungar, S.: The world of touch: an international survey of tactile maps. Part 2: design. British Journal of Visual Impairment. 21(3), 105-110 (2003) 
22. Simonnet, M.: Virtual reality contributions for the coordination of spatial frames of reference without vision. SeaTouch, a haptic and auditory application to set up the maritime itineraries of blind sailors. PhD Thesis. University of Brest (France), Department of Sports (2008)

23. Simonnet, M.: Sailing and blindness: The use of a sensorial perceptions system of a blind helmsman. Graduate Report, University of Brest (France), Department of Sports (2002)

24. Hardwick, D.A., McIntyre, C.W., Pick Jr, H.L.: The Content and Manipulation of Cognitive Maps in Children and Adults. Monographs of the Society for Research in Child Development. 41, 1-55 (1976)

25. Wang, R.F., Spelke, E.S.: Human spatial representation: insights from animals. Trends in Cognitive Sciences. 6, 376-382 (2002) 